\title{
Rapid Dermoscopic Changes in Nodular Melanoma
}

\author{
José Alberto García-Lozano, ${ }^{1}$ Gabriel Salerni, ${ }^{2}$ Adrian Cuellar-Barboza, ${ }^{1}$ \\ Jesús Alberto Cárdenas-de la Garza, ${ }^{1}$ Jorge Ocampo-Candiani ${ }^{1}$
}

1 Universidad Autónoma de Nuevo León, Servicio de Dermatología, Hospital Universitario Dr. José E. González, Monterrey, México 2 Hospital Provincial del Centenario de Rosario, Argentina, \& Facultad de Medicina, Universidad Nacional de Rosario, Argentina

Key words: dermoscopy, skin cancer, nodular melanoma, noninvasive imaging tools, dermato-oncology

Citation: García-Lozano JA, Salerni G, Cuellar-Barboza A, Cárdenas-de la Garza JA, Ocampo-Candiani J. Rapid dermoscopic changes in nodular melanoma. Dermatol Pract Concept. 2020;10(1):e2020016. DOI: https://doi.org/10.5826/dpc.1001a16

Accepted: August 29, 2019; Published: December 31, 2019

Copyright: @2019 García-Lozano et al. This is an open-access article distributed under the terms of the Creative Commons Attribution License, which permits unrestricted use, distribution, and reproduction in any medium, provided the original author and source are credited.

Funding: None.

Competing interests: The authors have no conflicts of interest to disclose.

Authorship: All authors have contributed significantly to this publication.

Corresponding author: Gabriel Salerni, MD, PhD, Bv. Oroño 1515, CP 2000, Rosario, Argentina. Email: gabrielsalerni@hotmail.com

\section{Introduction}

Nodular melanoma (NM) constitutes $9 \%$ to $15 \%$ of invasive melanoma and represents a potentially lethal skin tumor. It is the most frequent subtype of thick, rapidly growing melanomas and is often diagnosed at a locally advanced stage, conferring a worse prognosis [1]. In many countries, patients seek their first consultation with a general practitioner and then they are referred to a dermatologist. Other times, patients seek their first consultation with a dermatologist. Since it has been reported that NM can grow at a rate of $0.5 \mathrm{~mm}$ per month, time is crucial and even slight delays in the management of these lesions could affect the patient's prognosis.

\section{Case Presentation}

A 53-year-old woman with no history of melanoma presented with a lesion on her left lumbar region that had appeared 5 months previously. Physical examination revealed an erythematous, elevated, ulcerated nodular neoformation that was $10 \times 10 \mathrm{~mm}$ in diameter. Dermoscopy showed an asymmetric, achromic lesion with no specific criteria for melanocytic lesion; pink structureless central area, irregular blue blotches, and linear irregular vessels were observed (Figure 1A). Excision was scheduled for 10 days after the first consultation. Before the procedure, dermoscopy revealed significant changes: The lesion showed a prominent central crust, augmentation of blue-gray blotches, and significant increase in the irregular blood vessels at the periphery (Figure 1B). Histopathology reported an ulcerated NM, Breslow thickness $3.7 \mathrm{~mm}$, Clark IV with a mitotic rate of $5 / \mathrm{mm}^{2}$.

\section{Conclusions}

NM lesions may clinically present as symmetrical, firm papules or nodules with light color (amelanotic) and with regular border and therefore do not meet the "ABCD" rule (A for asymmetry, B for border irregularity, $\mathrm{C}$ for color variability, and $\mathrm{D}$ for diameter $>6 \mathrm{~mm}$ ). For this reason, the alternative "EFG" rule (E for elevation, $\mathrm{F}$ for firm consistency, and $\mathrm{G}$ for progressive growth) is more suitable for NM [1]. NM lesions grow rapidly $(0.5 \mathrm{~mm} / \mathrm{month})$, develop depth of invasion faster than other radial growth melanomas, and account for $66 \%$ of tumors thicker than $3 \mathrm{~mm}$; for these reasons, NM causes a high percentage of deaths due to melanoma [2]. In the present case, within a 10-day interval between first 
consultation and surgical procedure, we observed by dermoscopic examination that the lesion underwent significant changes, especially an increase in vascularization.

Taking this case into consideration, we encourage dermatologists to take urgent action when NM cannot be ruled out to avoid unnecessary delay that could affect the patient's prognosis. More population-based strategies to increase awareness about risk factors and clinical features are needed to detect NM at an early stage since classic skin cancer screening campaigns are unlikely to improve early diagnosis of this melanoma subtype.

\section{References}

1. Menzies SW, Moloney FJ, Byth K, et al. Dermoscopic evaluation of nodular melanoma. JAMA Dermatol. 2013;149(6):699709.

2. Kelly JW, Chamberlain AJ, Staples MP, McAvoy B. Nodular melanoma: no longer as simple as ABC. Aust Fam Physician. 2003;32(9):706-709.
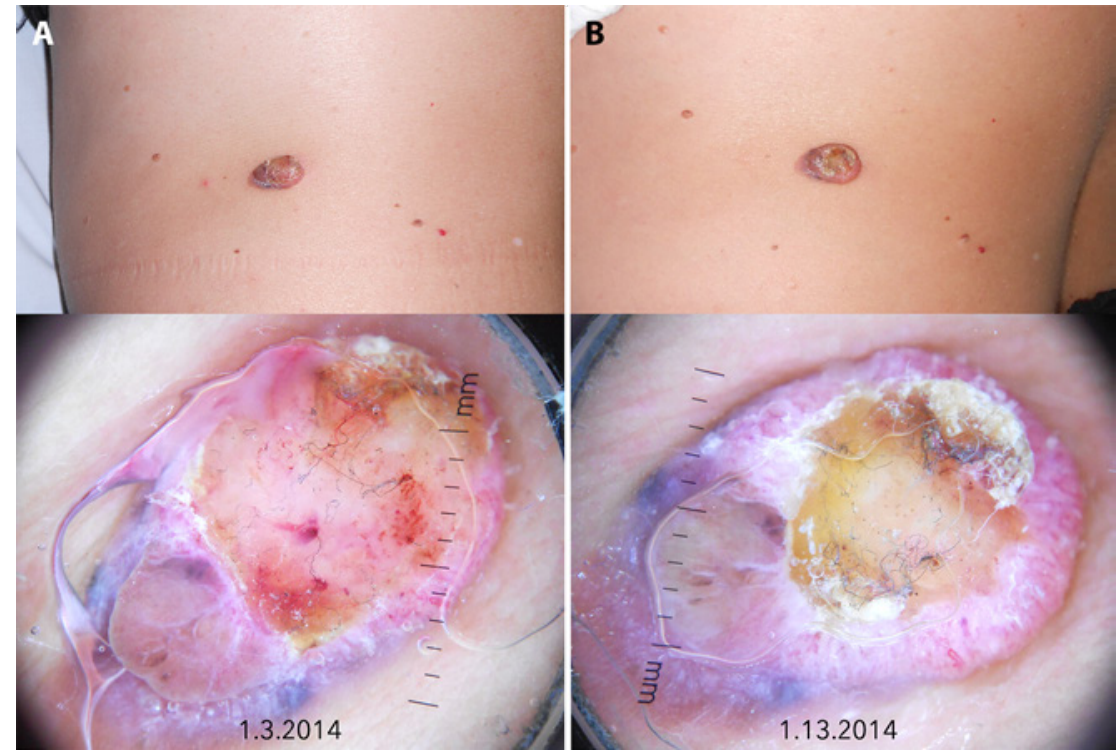

Figure 1. Clinical and dermoscopic image of the ulcerated nodular melanoma on the first consultation (A) and before surgical procedure (B). Note the significant changes in the lesion within 10 days. 